\title{
BOUNDEDNESS IN PERTURBED NONLINEAR DIFFERENTIAL SYSTEMS
}

\author{
Yoon Hoe Goo*
}

\begin{abstract}
In this paper, we investigate bounds for solutions of perturbed nonlinear differential systems.
\end{abstract}

\section{Introduction}

The behavior of solutions of a perturbed system is determined in terms of the behavior of solutions of an unperturbed system. There are three useful methods for showing the qualitative behavior of the solutions of perturbed nonlinear system : Lyapunov's second method, the method of variation of constants formula, and the use of inequalities.

The notion of $h$-stability (hS) was introduced by Pinto [11, 12] with the intention of obtaining results about stability for a weakly stable system (at least, weaker than those given exponential asymptotic stability) under some perturbations. That is, Pinto extended the study of exponential asymptotic stability to a variety of reasonable systems called $h$-systems.

The main conclusion to be drawn from this paper is that the use of inequalities provides a powerful tool for obtaining bounds for solutions. To do this we need some integral inequalities.

\section{Preliminaries}

We consider the nonlinear nonautonomous differential system

$$
x^{\prime}(t)=f(t, x(t)), \quad x\left(t_{0}\right)=x_{0},
$$

where $f \in C\left(\mathbb{R}^{+} \times \mathbb{R}^{n}, \mathbb{R}^{n}\right), \mathbb{R}^{+}=[0, \infty)$ and $\mathbb{R}^{n}$ is the Euclidean $n$ space. We assume that the Jacobian matrix $f_{x}=\partial f / \partial x$ exists and

Received May 28, 2013; Accepted July 18, 2013.

2010 Mathematics Subject Classification: Primary 34D10.

Key words and phrases: $h$-stability, $t_{\infty}$-similarity, nonlinear nonautonomous system. 
is continuous on $\mathbb{R}^{+} \times \mathbb{R}^{n}$ and $f(t, 0)=0$. For $x \in \mathbb{R}^{n}$, let $|x|=$ $\left(\sum_{j=1}^{n} x_{j}^{2}\right)^{1 / 2}$. For an $n \times n$ matrix $A$, define the norm $|A|$ of $A$ by $|A|=\sup _{|x| \leq 1}|A x|$.

Let $x\left(t, t_{0}, x_{0}\right)$ denote the unique solution of $(2.1)$ with $x\left(t_{0}, t_{0}, x_{0}\right)=$ $x_{0}$, existing on $\left[t_{0}, \infty\right)$. Then we can consider the associated variational systems around the zero solution of (2.1) and around $x(t)$, respectively,

$$
v^{\prime}(t)=f_{x}(t, 0) v(t), v\left(t_{0}\right)=v_{0}
$$

and

$$
z^{\prime}(t)=f_{x}\left(t, x\left(t, t_{0}, x_{0}\right)\right) z(t), z\left(t_{0}\right)=z_{0} .
$$

The fundamental matrix $\Phi\left(t, t_{0}, x_{0}\right)$ of $(2.3)$ is given by

$$
\Phi\left(t, t_{0}, x_{0}\right)=\frac{\partial}{\partial x_{0}} x\left(t, t_{0}, x_{0}\right)
$$

and $\Phi\left(t, t_{0}, 0\right)$ is the fundamental matrix of $(2.2)$.

We recall some notions of $h$-stability [12].

Definition 2.1. The system (2.1)(the zero solution $x=0$ of (2.1)) is called an $h$-system if there exist a constant $c \geq 1$, and a positive continuous function $h$ on $\mathbb{R}^{+}$such that

$$
|x(t)| \leq c\left|x_{0}\right| h(t) h\left(t_{0}\right)^{-1}
$$

for $t \geq t_{0} \geq 0$ and $\left|x_{0}\right| \operatorname{small}$ enough(here $\left.h(t)^{-1}=\frac{1}{h(t)}\right)$.

Definition 2.2. The system (2.1) (the zero solution $x=0$ of (2.1)) is called $h$-stable $(h S)$ if there exists $\delta>0$ such that (2.1) is an $h$-system for $\left|x_{0}\right| \leq \delta$ and $h$ is bounded.

Let $\mathcal{M}$ denote the set of all $n \times n$ continuous matrices $A(t)$ defined on $\mathbb{R}^{+}$and $\mathcal{N}$ be the subset of $\mathcal{M}$ consisting of those nonsingular matrices $S(t)$ that are of class $C^{1}$ with the property that $S(t)$ and $S^{-1}(t)$ are bounded. The notion of $t_{\infty}$-similarity in $\mathcal{M}$ was introduced by Conti [6].

Definition 2.3. A matrix $A(t) \in \mathcal{M}$ is $t_{\infty}$-similar to a matrix $B(t) \in$ $\mathcal{M}$ if there exists an $n \times n$ matrix $F(t)$ absolutely integrable over $\mathbb{R}^{+}$, i.e.,

$$
\int_{0}^{\infty}|F(t)| d t<\infty
$$

such that

$$
\dot{S}(t)+S(t) B(t)-A(t) S(t)=F(t)
$$

for some $S(t) \in \mathcal{N}$. 
The notion of $t_{\infty}$-similarity is an equivalence relation in the set of all $n \times n$ continuous matrices on $\mathbb{R}^{+}$, and it preserves some stability concepts [6].

We give some related properties that we need in the sequal.

Lemma 2.4. [13] The linear system

$$
x^{\prime}=A(t) x, x\left(t_{0}\right)=x_{0},
$$

where $A(t)$ is an $n \times n$ continuous matrix, is an $h$-system(respectively $h$ stable) if and only if there exist $c \geq 1$ and a positive continuous(repectively bounded) function $h$ defined on $\mathbb{R}^{+}$such that

$$
\left|\phi\left(t, t_{0}\right)\right| \leq c h(t) h\left(t_{0}\right)^{-1}
$$

for $t \geq t_{0} \geq 0$, where $\phi\left(t, t_{0}\right)$ is a fundamental matrix of (2.5).

We need Alekseev formula to compare between the solutions of (2.1) and the solutions of perturbed nonlinear system

$$
y^{\prime}=f(t, y)+g(t, y), y\left(t_{0}\right)=y_{0},
$$

where $g \in C\left(\mathbb{R}^{+} \times \mathbb{R}^{n}, \mathbb{R}^{n}\right)$ and $g(t, 0)=0$. Let $y(t)=y\left(t, t_{0}, y_{0}\right)$ denote the solution of (2.7) passing through the point $\left(t_{0}, y_{0}\right)$ in $\mathbb{R}^{+} \times \mathbb{R}^{n}$.

The following is a generalization to nonlinear system of the variation of constants formula due to Alekseev [1].

Lemma 2.5. If $y_{0} \in \mathbb{R}^{n}$, then for all $t$ such that $x\left(t, t_{0}, y_{0}\right) \in \mathbb{R}^{n}$,

$$
y\left(t, t_{0}, y_{0}\right)=x\left(t, t_{0}, y_{0}\right)+\int_{t_{0}}^{t} \Phi(t, s, y(s)) g(s, y(s)) d s .
$$

TheOREM 2.6. [3] If the zero solution of (2.1) is $h S$, then the zero solution of (2.2) is $h S$.

Theorem 2.7. [4] Suppose that $f_{x}(t, 0)$ is $t_{\infty}$-similar to $f_{x}\left(t, x\left(t, t_{0}, x_{0}\right)\right)$ for $t \geq t_{0} \geq 0$ and $\left|x_{0}\right| \leq \delta$ for some constant $\delta>0$. If the solution $v=0$ of (2.2) is hS, then the solution $z=0$ of (2.3) is $h S$.

Lemma 2.8. (Bihari-type inequality) Let $u, \lambda \in C\left(\mathbb{R}^{+}\right), w \in C((0, \infty))$ and $w(u)$ be nondecreasing in $u$. Suppose that, for some $c>0$,

$$
u(t) \leq c+\int_{t_{0}}^{t} \lambda(s) w(u(s)) d s, t \geq t_{0} \geq 0 .
$$

Then

$$
u(t) \leq W^{-1}\left[W(c)+\int_{t_{0}}^{t} \lambda(s) d s\right], t_{0} \leq t<b_{1},
$$


where $W(u)=\int_{u_{0}}^{u} \frac{d s}{w(s)}, W^{-1}(u)$ is the inverse of $W(u)$ and

$$
b_{1}=\sup \left\{t \geq t_{0}: W(c)+\int_{t_{0}}^{t} \lambda(s) d s \in \operatorname{domW}^{-1}\right\} .
$$

Lemma 2.9. [2] Let $u, \lambda_{1}, \lambda_{2}, \lambda_{3} \in C\left(\mathbb{R}^{+}\right), w \in C((0, \infty))$ and $w(u)$ be nondecreasing in $u$. Suppose that for some $c>0$,

$u(t) \leq c+\int_{t_{0}}^{t} \lambda_{1}(s) w(u(s)) d s+\int_{t_{0}}^{t} \lambda_{2}(s)\left(\int_{t_{0}}^{s} \lambda_{3}(\tau) w(u(\tau)) d \tau\right) d s, 0 \leq t_{0} \leq t$.

Then

$$
u(t) \leq W^{-1}\left[W(c)+\int_{t_{0}}^{t}\left(\lambda_{1}(s)+\lambda_{2}(s) \int_{t_{0}}^{s} \lambda_{3}(\tau)\right) d s\right], t_{0} \leq t<b_{1},
$$

where $W(u)=\int_{u_{0}}^{u} \frac{d s}{w(s)}, u>0, u_{0}>0, W^{-1}(u)$ is the inverse of $W(u)$ and

$$
b_{1}=\sup \left\{t \geq t_{0}: W(c)+\int_{t_{0}}^{t}\left(\lambda_{1}(s)+\lambda_{2}(s) \int_{t_{0}}^{s} \lambda_{3}(\tau)\right) d s \in \operatorname{domW}^{-1}\right\} .
$$

\section{Main results}

In this section, we investigate bounds for the nonlinear differential systems.

TheOREM 3.1. Let $a, k, u, w \in C\left(\mathbb{R}^{+}\right), w(u)$ be nondecreasing in $u$ and $\frac{1}{v} w(u) \leq w\left(\frac{u}{v}\right)$ for some $v>0$. Suppose that the solution $x=0$ of (2.1) is $h S$ with a nondecreasing function $h$ and the perturbed term $g$ in (2.7) satisfies

$$
|\Phi(t, s, z) g(t, z)| \leq a(s)\left(w(|z|)+\int_{t_{0}}^{s} k(\tau) w(|z|) d \tau\right), t \geq t_{0} \geq 0,
$$

where $\int_{t_{0}}^{\infty} k(s) d s<\infty$. Then any solution $y(t)=y\left(t, t_{0}, y_{0}\right)$ of $(2.7)$ is bounded on $\left[t_{0}, \infty\right)$ and it satisfies

$$
|y(t)| \leq h(t) W^{-1}\left[W(c)+\int_{t_{0}}^{t} a(s)\left(1+\int_{t_{0}}^{s} k(\tau) d \tau\right) d s\right], t_{0} \leq t<b_{1},
$$

where $W, W^{-1}$ are the same functions as in Lemma 2.8 and

$$
b_{1}=\sup \left\{t \geq t_{0}: W(c)+\int_{t_{0}}^{t} a(s)\left(1+\int_{t_{0}}^{s} k(\tau) d \tau\right) d s \in \operatorname{domW}^{-1}\right\} .
$$


Proof. Let $x(t)=x\left(t, t_{0}, y_{0}\right)$ and $y(t)=y\left(t, t_{0}, y_{0}\right)$ be solutions of (2.1) and (2.7), respectively. Applying Lemma 2.5 and the increasing property of the function $h$, we obtain

$$
\begin{aligned}
|y(t)| \leq & |x(t)|+\int_{t_{0}}^{t}|\Phi(t, s, y(s)) g(s, y(s))| d s \\
\leq & c_{1}\left|y_{0}\right| h(t) h\left(t_{0}\right)^{-1}+\int_{t_{0}}^{t} a(s)\left[w(|y(s)|)+\int_{t_{0}}^{s} k(\tau) w(|y(\tau)|) d \tau\right] d s \\
\leq & c_{1}\left|y_{0}\right| h(t) h\left(t_{0}\right)^{-1}+\int_{t_{0}}^{t} a(s) h(t) w\left(\frac{|y(s)|}{h(s)}\right) d s \\
& \quad+\int_{t_{0}}^{t} a(s) \int_{t_{0}}^{s} h(t) k(\tau) w\left(\frac{|y(\tau)|}{h(\tau)}\right) d \tau d s .
\end{aligned}
$$

Set $u(t)=|y(t)| h(t)^{-1}$. Then, by Lemma 2.9, we have

$$
|y(t)| \leq h(t) W^{-1}\left[W(c)+\int_{t_{0}}^{t} a(s)\left(1+\int_{t_{0}}^{s} k(\tau) d \tau\right], t_{0} \leq t<b_{1},\right.
$$

where $c=c_{1}\left|y_{0}\right| h\left(t_{0}\right)^{-1}$. The above estimation yields the desired result since the function $h$ is bounded, and the theorem is proved.

REMARK 3.1. Letting $k(t)=0$ in Theorem 3.1, we obtain the same result as that of Theorem 3.1 in [9].

Also, we examine the bounded property for the perturbed system

$$
y^{\prime}=f(t, y)+\int_{t_{0}}^{t} g(s, y(s)) d s, y\left(t_{0}\right)=y_{0},
$$

where $g \in C\left(\mathbb{R}^{+} \times \mathbb{R}^{n}, \mathbb{R}^{n}\right)$ and $g(t, 0)=0$, of $(2.1)$.

TheOrem 3.2. Let $a, b, k, u, w \in C\left(\mathbb{R}^{+}\right), w(u)$ be nondecreasing in $u$ and $\frac{1}{v} w(u) \leq w\left(\frac{u}{v}\right)$ for some $v>0$. Suppose that $f_{x}(t, 0)$ is $t_{\infty}$-similar to $f_{x}\left(t, x\left(t, t_{0}, x_{0}\right)\right)$ for $t \geq t_{0} \geq 0$ and $\left|x_{0}\right| \leq \delta$ for some constant $\delta>0$, the solution $x=0$ of (2.1) is $h S$ with the increasing function $h$, and $g$ in (3.1) satisfies

$$
\left|\int_{t_{0}}^{s} g(\tau, y(\tau)) d \tau\right| \leq a(s) w(|y(s)|)+b(s) \int_{t_{0}}^{s} k(\tau) w(|y(\tau)|) d \tau,
$$

where $\int_{t_{0}}^{\infty} a(s) d s<\infty, \int_{t_{0}}^{\infty} b(s) d s<\infty$ and $\int_{t_{0}}^{\infty} k(s) d s<\infty$. Then, any solution $y(t)=y\left(t, t_{0}, y_{0}\right)$ of (3.1) is bounded on $\left[t_{0}, \infty\right)$ and it satisfies

$$
|y(t)| \leq h(t) W^{-1}\left[W(c)+c_{2} \int_{t_{0}}^{t}\left(a(s)+b(s) \int_{t_{0}}^{s} k(\tau) d \tau\right) d s\right],
$$


where $W, W^{-1}$ are the same functions as in Lemma 2.8 and

$$
b_{1}=\sup \left\{t \geq t_{0}: W(c)+c_{2} \int_{t_{0}}^{t}\left(a(s)+b(s) \int_{t_{0}}^{s} k(\tau) d \tau\right) d s \in \operatorname{domW}^{-1}\right\} .
$$

Proof. Let $x(t)=x\left(t, t_{0}, x_{0}\right)$ and $y(t)=y\left(t, t_{0}, x_{0}\right)$ be solutions of (2.1) and (3.1), respectively. By Theorem 2.6, since the solution $x=0$ of $(2.1)$ is $\mathrm{hS}$, the solution $v=0$ of $(2.2)$ is $\mathrm{hS}$. Therefore, by Theorem 2.7 , the solution $z=0$ of (2.3) is hS. Using two Lemma 2.4 and 2.5, we have

$$
\begin{gathered}
|y(t)| \leq|x(t)|+\int_{t_{0}}^{t}|\Phi(t, s, y(s))|\left|\int_{t_{0}}^{s} g(\tau, y(\tau)) d \tau\right| d s \\
\leq c_{1}\left|y_{0}\right| h(t) h\left(t_{0}\right)^{-1}+\int_{t_{0}}^{t} c_{2} h(t) a(s) w\left(\frac{|y(s)|}{h(s)}\right) d s \\
\quad+\int_{t_{0}}^{t} c_{2} h(t) b(s) \int_{t_{0}}^{s} k(\tau) w\left(\frac{|y(\tau)|}{h(\tau)}\right) d \tau d s .
\end{gathered}
$$

since $h$ is increasing. Set $u(t)=|y(t)| h(t)^{-1}$. Now an application of Lemma 2.9 yields

$$
|y(t)| \leq h(t) W^{-1}\left[W(c)+c_{2} \int_{t_{0}}^{t}\left(a(s)+b(s) \int_{t_{0}}^{s} k(\tau) d \tau\right) d s\right]
$$

where $c=c_{1}\left|y_{0}\right| h\left(t_{0}\right)^{-1}$. The above estimation implies the boundedness of $y(t)$, and the proof is complete.

Remark 3.2. Letting $k(t)=0$ in Theorem 3.2, we obtain the same result as that of Theorem 3.2 in [9].

We need the lemma to prove the following theorem.

Lemma 3.3. Let $u, p, q, w, r \in C\left(\mathbb{R}^{+}\right), w \in C((0, \infty))$ and $w(u)$ be nondecreasing in $u$ and $u \leq w(u)$. Suppose that for some $c \geq 0$,

$u(t) \leq c+\int_{t_{0}}^{t}\left(p(s) \int_{t_{0}}^{s}\left(q(\tau) u(\tau)+v(\tau) \int_{t_{0}}^{\tau} r(a) w(u(a)) d a\right) d \tau\right) d s, t \geq t_{0}$.

Then

$u(t) \leq W^{-1}\left[W(c)+\int_{t_{0}}^{t}\left(p(s) \int_{t_{0}}^{s}\left(q(\tau)+v(\tau) \int_{t_{0}}^{\tau} r(a) d a\right) d \tau\right) d s\right], t_{0} \leq t<b_{1}$, 
where $W(u)=\int_{u_{0}}^{u} \frac{d s}{w(s)}, W^{-1}(u)$ is the inverse of $W(u)$ and

$$
\begin{aligned}
b_{1}=\sup \left\{t \geq t_{0}: W(c)+\int_{t_{0}}^{t}\right. & \left(p(s) \int_{t_{0}}^{s}(q(\tau)\right. \\
& \left.\left.\left.+v(\tau) \int_{t_{0}}^{\tau} r(a) d a\right) d \tau\right) d s \in \mathrm{domW}^{-1}\right\} .
\end{aligned}
$$

Proof. Setting

$$
z(t)=c+\int_{t_{0}}^{t}\left(p(s) \int_{t_{0}}^{s}\left(q(\tau) u(\tau)+v(\tau) \int_{t_{0}}^{\tau} r(a) w(u(a)) d a\right) d \tau\right) d s,
$$

we have $z\left(t_{0}\right)=c$ and

$$
\begin{aligned}
z^{\prime}(t) & =p(t) \int_{t_{0}}^{t}\left(q(\tau) u(\tau)+v(\tau) \int_{t_{0}}^{\tau} r(a) w(u(a)) d a\right) d \tau \\
& \leq p(t) \int_{t_{0}}^{t}\left(q(\tau)+v(\tau) \int_{t_{0}}^{\tau} r(a) d a\right) w(u(\tau)) d \tau \\
& \leq\left[p(t) \int_{t_{0}}^{t}\left(q(\tau)+v(\tau) \int_{t_{0}}^{\tau} r(a) d a\right) d \tau\right] w(z(t)), t \geq t_{0},
\end{aligned}
$$

since $z(t)$ and $w(u)$ are nondecreasing and $u(t) \leq z(t)$. Therefore, by integrating on $\left[t_{0}, t\right]$, the function $z$ satisfies

$$
z(t) \leq c+\int_{t_{0}}^{t}\left(p(s) \int_{t_{0}}^{s}\left(q(\tau)+v(\tau) \int_{t_{0}}^{\tau} r(a) d a\right) d \tau w(z(s))\right) d s .
$$

It follows from Lemma 2.8 that (3.5) yields the estimate (3.3).

Theorem 3.4. Let $w \in C\left(\mathbb{R}^{+}\right), w(u)$ be nondeacreasing in $u, u \leq$ $w(u)$, and $\frac{1}{v} w(u) \leq w\left(\frac{u}{v}\right)$ for some $v>0$. Suppose that $f_{x}(t, 0)$ is $t_{\infty^{-}}$ similar to $f_{x}\left(t, x\left(t, t_{0}, x_{0}\right)\right)$ for $t \geq t_{0} \geq 0$ and $\left|x_{0}\right| \leq \delta$ for some constant $\delta>0$. If the solution $x=0$ of (2.1)is an h-system with a positive continuous function $h$ and $g$ in (3.1) satisfies

$$
|g(t, y)| \leq a(t)\left(|y(t)|+\int_{t_{0}}^{t} k(s) w(|y(s)|) d s\right), t \geq t_{0}, y \in \mathbb{R}^{n}
$$

where $a: \mathbb{R}^{+} \rightarrow \mathbb{R}^{+}$is continuous with

$$
\int_{t_{0}}^{\infty} \frac{1}{h(s)} \int_{t_{0}}^{s}\left(a(\tau)\left(h(\tau)+\int_{t_{0}}^{\tau} h(r) k(r) d r\right) d \tau d s<\infty,\right.
$$

for all $t_{0} \geq 0$, then any solution $y(t)=y\left(t, t_{0}, y_{0}\right)$ of (3.1) satisfies

$$
|y(t)| \leq h(t) W^{-1}\left[W(c)+\int_{t_{0}}^{t} \frac{c_{2}}{h(s)} \int_{t_{0}}^{s} a(\tau)\left(h(\tau)+\int_{t_{0}}^{\tau} h(r) k(r) d r\right) d \tau d s\right],
$$


$t_{0} \leq t<b_{1}$, where $W, W^{-1}$ are the same functions as in Lemma 2.8 and

$$
\begin{aligned}
b_{1}=\sup \left\{t \geq t_{0}: W(c)+\int_{t_{0}}^{t} \frac{c_{2}}{h(s)} \int_{t_{0}}^{s} a(\tau)(h(\tau)\right. \\
\left.\left.\quad+\int_{t_{0}}^{\tau} h(r) k(r) d r\right) d \tau d s \in \text { domW }^{-1}\right\} .
\end{aligned}
$$

Proof. Let $x(t)=x\left(t, t_{0}, x_{0}\right)$ and $y(t)=y\left(t, t_{0}, x_{0}\right)$ be solutions of (2.1) and (3.1), respectively. By Theorem 2.6, since the solution $x=0$ of (2.1) is an h-system, the solution $v=0$ of (2.2) is an h-system. Therefore, by Theorem 2.7, the solution $z=0$ of (2.3) is an h-system. Applying Lemma 2.5 and (3.6), we have

$$
\begin{gathered}
|y(t)| \leq|x(t)|+\int_{t_{0}}^{t}|\Phi(t, s, y(s))| \int_{t_{0}}^{s}|g(\tau, y(\tau))| d \tau d s \\
\leq c_{1}\left|y_{0}\right| h(t) h\left(t_{0}\right)^{-1}+\int_{t_{0}}^{t} c_{2} \frac{h(t)}{h(s)}\left(\int_{t_{0}}^{s} h(\tau) a(\tau) \frac{|y(\tau)|}{h(\tau)}\right. \\
\left.\quad+\int_{t_{0}}^{s} a(\tau) \int_{t_{0}}^{\tau} h(r) k(r) w\left(\frac{y(r) \mid}{h(r)}\right) d r\right) d \tau d s .
\end{gathered}
$$

Using Lemma 3.3 with $u(t)=|y(t)| h(t)^{-1}$, we obtain

$|y(t)| \leq h(t) W^{-1}\left[W(c)+\int_{t_{0}}^{t} \frac{c_{2}}{h(s)} \int_{t_{0}}^{s} a(\tau)\left(h(\tau)+\int_{t_{0}}^{\tau} h(r) k(r) d r\right) d \tau d s\right]$,

$t_{0} \leq t<b_{1}$, where $c=c_{1}\left|y_{0}\right| h\left(t_{0}\right)^{-1}$. Hence, the proof is complete.

Remark 3.3. Letting $k(s)=0$ in Theorem 3.4, we obtain the same result as that of Theorem 2.5 in [10]

\section{Acknowledgments}

The author is very grateful for the referee's valuable comments.

\section{References}

[1] F. Brauer, Perturbations of nonlinear systems of differential equations II, J. Math. Anal. Appl. 17 (1967), 579-591.

[2] S. K. Choi and N. J. Koo, h-stability for nonlinear perturbed systems, Ann. of Diff. Eqs. 11 (1995), 1-9.

[3] S. K. Choi and H. S. Ryu, h-stability in differential systems, Bull. Inst. Math. Acad. Sinica 21 (1993), 245-262. 
[4] S. K. Choi, N. J. Koo, and H. S. Ryu, h-stability of differential systems via $t_{\infty}$-similarity, Bull. Korean. Math. Soc. 34 (1997), 371-383.

[5] S. K. Choi, N. J. Koo, and S. M. Song, Lipschitz stability for nonlinear functional differential systems, Far East J. Math. Sci(FJMS)I 5 (1999), 689-708.

[6] R. Conti, Sulla $t_{\infty}$-similitudine tra matricie l'equivalenza asintotica dei sistemi differenziali lineari, Rivista di Mat. Univ. Parma 8 (1957), 43-47.

[7] S. Elaydi and R. R. M. Rao, Lipschitz stability for nonlinear Volterra integrodifferential systems, Appl. Math. Computations 27 (1988), 191-199.

[8] P. Gonzalez and M. Pinto, Stability properties of the solutions of the nonlinear functional differential systems, J. Math. Appal. 182 (1994), 562-573.

[9] Y. H. Goo, D. G. Park, and D. H. Ryu, Boundedness in perturbed differential systems, J. Appl. Math. and Informatics 30 (2012),279-287.

[10] Y . H. Goo, h-stability of the nonlinear differential systems, J. Chungcheong Math. Soc. 23 (2010), 383-389.

[11] V. Lakshmikantham and S. Leela, Differential and Integral Inequalities: Theory and Applications Vol. I, Academic Press, New York and London, 1969.

[12] M. Pinto, Perturbations of asymptotically stable differential systems, Analysis 4 (1984), 161-175.

[13] M. Pinto, Stability of nonlinear differential systems, Applicable Analysis 43 (1992), 1-20.

[14] M. R. M. Rao and P. Srinivas, Asymptotic behavior of solutions of Volterra integro-differential equations, Proc. Amer. Math. Soc. 94 (1985), 55-60.

$*$

Department of Mathematics

Hanseo University

Chungnam 356-706, Republic of Korea

E-mail: yhgoo@hanseo.ac.kr 